\title{
Intervenções não farmacológicas na melhoria da qualidade de vida de crianças/adolescentes oncológicos
} Nonpharmacological interventions in the improvement of quality of

life in children and adolescent cancer patients Intervenciones no farmacológicas en la mejora de la calidad de vida de niños/adolescentes oncológicos

Welker da Silva Xavier (1D https://orcid.org/0000-0001-7495-30051 Sandra Teixeira de Araújo Pacheco it https://orid.org/0000-0002-4612-889x²

Liliane Faria da Silva io hitps://orcid.org/0000-0002-9125-1053

Lucila Castanheira Nascimento io https://orcid.org/0000-0002-7900-71114

Luís Carlos Lopes-Junior io https://orid.org/0000-0002-2424-65105 Barbara Bertolossi Marta de Araújo io hitps://orcid.org//0000-0001-9421-01612 Michelle Darezzo Rodrigues Nunes io https://orid.org/0000-0001-7685-342X2

\section{Como citar:}

Xavier WS, Pacheco ST, Silva LF, Nascimento LC, Lopes-Júnior LC, Araújo BB, et al. Intervenções não farmacológicas na melhoria da qualidade de vida de crianças/adolescentes oncológicos. Acta Paul Enferm. 2020;33:e-APE-20190022

DOI

http://dx.doi.org/10.37689/ acta-ape/2020AR0022

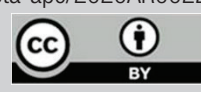

Descritores

Criança; Adolescente; Neoplasias; Qualidade de vida; Enfermagem pediátrica

Keywords

Child; Adolescente; Neoplasms; Quality of life: Pediatric nursing

Descriptores

Niño; Adolescente; Neoplasias; Calidad de vida;

Enfermería pediátrica

Submetido 29 de Janeiro de 2019

Aceito

14 de Agosto de 2019

Autor correspondente

Michelle Darezzo Rodrigues Nunes https://orcid.org/0000-0001-7685-342X Email: mid13@hotmail.com

\section{Resumo}

Objetivo: Identificar estudos na literatura nacional e internacional sobre a eficácia das intervenções não farmacológicas para melhoria da qualidade de vida de crianças e adolescentes com câncer.

Métodos: Revisão integrativa, cuja busca ocorreu nos meses de setembro e outubro de 2018, nas bases de dados PsycINFO, Web of Science, CINAHL, LILACS, IBECS, BDENF e MEDLINE® para responder à questão norteadora: "Quais são as intervenções não farmacológicas disponíveis na literatura e utilizadas para melhorar a qualidade de vida de crianças e adolescentes com câncer?". Foram incluídos artigos originais, publicados entre 2013 e 2018, nos idiomas inglês, português ou espanhol. Excluíram-se estudos que focavam outras temáticas, população adulta, artigos de revisão, dissertações e teses.

Resultados: A amostra foi constituída por 22 artigos, que foram agrupados, conforme suas similaridades, em três categorias. As intervenções utilizadas foram: atividade física exclusiva (6); atividade física conciliada à outra intervenção (8); e intervenções psicológicas (8). Destas intervenções, 13 apresentaram diferenças significativas na melhoria da qualidade de vida.

Conclusão: As intervenções não farmacológicas utilizando exercício físico exclusivo ou conciliado a outra intervenção foram as mais eficazes. Esta revisão auxilia na sensibilização dos profissionais sobre a importância da temática e fornece subsídios para o planejamento de ações estratégicas, no âmbito da enfermagem pediátrica, que incluam intervenções não farmacológicas, conciliadas ao tratamento padrão, para a melhoria da qualidade de vida de crianças e adolescentes com câncer. Os resultados devem ser avaliados com cautela, pois identificou-se uma diversidade de intervenções, protocolos e número de participantes - 0 que dificulta a generalização dos achados.

\section{Abstract}

Objective: To identify, in both the Brazilian and international literature, studies about the efficacy of nonpharmacological interventions oriented toward improving quality of life in children and adolescents with cancer.

Methods: Integrative review whose search process was carried out in September and October 2018 in the following databases: PsycINFO, Web of Science, Cumulative Index to Nursing and Allied Health Literature, Latin America and Caribbean Center on Health Sciences Information, Índice Bibliográfico Español de Ciencias de la Salud, Banco de Dados em Enfermagem, and MEDLINE $®$. The guiding question was "What are the nonpharmacological interventions available in the literature used to improve quality of life in children and

Instituto Nacional de Saúde da Mulher, da Criança e do Adolescente Rio de Janeiro, RJ Brasil.

${ }^{2}$ Faculdade de Enfermagem, Universidade do Estado do Rio de Janeiro, Rio de Janeiro, RJ, Brasil.

${ }^{3}$ Escola de Enfermagem Aurora de Afonso Costa, Universidade Federal Fluminense, Niterói, RJ, Brasil.

${ }^{4}$ Escola de Enfermagem de Ribeirão Preto, Universidade de São Paulo, Ribeirão Preto, SP, Brasil.

Enniversidade Federal do Espírito Santo, Vitória, ES, Brasil.

Conflitos de interesse: nada a declarar. 
adolescents with cancer?". Original articles published between 2013 and 2018 in English, Portuguese, or Spanish were included. Studies that focused on other subjects or adult population and review articles, dissertations, and theses were excluded.

Results: The sample was 22 articles, which were grouped, according to their similarities, into three categories. The described interventions consisted of: exclusive physical activity (6); physical activity combined with another intervention (8); and psychological interventions (8). Among these interventions, 13 showed significant differences in the improvement of quality of life.

Conclusion: The nonpharmacological interventions that applied physical exercise or were combined with another intervention were the most effective. The present review helps raising awareness of professionals of the importance of the subject and provides resources to plan strategical actions, in the context of pediatric nursing, that include nonpharmacological interventions, together with the standard treatment, to improve quality of life in children and adolescents with cancer. The results must be evaluated with caution, given that a variety of interventions, protocols, and number of participants was identified in the sample, which hinders the generalization of the findings.

\section{Resumen}

Objetivo: Identificar estudios en la literatura nacional e internacional sobre la eficacia de las intervenciones no farmacológicas en la mejora de la calidad de vida de niños y adolescentes con cáncer.

Métodos: Revisión integradora, cuya búsqueda ocurrió en los meses de septiembre y octubre de 2018, en las bases de datos PsycINF0, Web of Science, CINAHL, LILACS, IBECS, BDENF y MEDLINE® para responder la pregunta orientadora: “¿Cuáles son las intervenciones no farmacológicas disponibles en la literatura y utilizadas para mejorar la calidad de vida de niños y adolescentes con cáncer?". Se incluyeron artículos originales publicados entre 2013 y 2018 en los idiomas inglés, portugués y español. Se excluyeron estudios que se centraban en otras temáticas, como población adulta, artículos de revisión, tesis de maestría y doctorado.

Resultados: La muestra fue formada por 22 artículos, que fueron agrupados en tres categorías según sus similitudes. Las intervenciones utilizadas fueron: actividad física exclusiva (6), actividad física conciliada con otra intervención (8) e intervenciones psicológicas (8). De estas intervenciones, 13 presentaron diferencias significativas en la mejora de la calidad de vida.

Conclusión: Las intervenciones no farmacológicas utilizando ejercicio físico exclusivo o conciliado con otra intervención fueron las más eficaces. Esta revisión ayuda a sensibilizar a los profesionales sobre la importancia de esta temática y contribuye a la planificación de acciones estratégicas en el ámbito de la enfermería pediátrica, que incluyan intervenciones no farmacológicas, conciliadas con el tratamiento estándar, para la mejora de la calidad de vida de niños y adolescentes con cáncer. Los resultados deben analizarse con cautela, ya que se identificó una diversidad de intervenciones, protocolos y número de participantes, lo que dificulta la generalización de los descubrimientos.

\section{Introdução}

O câncer é uma condição crônica, considerada um problema de saúde pública mundial. Sua alta prevalência, a relevância como causa de morte, o elevado consumo de recursos financeiros e o ônus psicossocial têm reunido esforços para implementar açôes e programas para seu controle e sua prevenção. ${ }^{(1)}$

O câncer infantil, especificamente, caracterizase como um conjunto de diferentes malignidades, variando em relação à histopatologia e ao comportamento clínico. ${ }^{(1)}$ No Brasil, o câncer representa a segunda causa de morte de crianças, adolescentes e adultos jovens, sendo que a faixa etária de 15 a 19 anos apresenta maior risco. $\mathrm{O}$ percentual mediado de neoplasias na população infantojuvenil $(0$ a 19 anos) é de $3 \%$, sendo observada maior frequência de leucemias, linfomas e tumores de sistema nervoso central. ${ }^{(1)}$

Adoecer de câncer promove grande impacto na vida de crianças, adolescentes e seus familiares, significando conviver com sinais e sintomas que surgem repentinamente e idas frequentes a hospi- tais, além de grandes alteraçôes em suas rotinas. ${ }^{(2,3)}$ Durante o tratamento oncológico, os pacientes são submetidos a internaçóes longas para realização de exames e tratamentos que envolvem quimioterapia, radioterapia, cirurgia e utilização de diversos medicamentos que, em conjunto, geram limitaçôes físicas e psicológicas ${ }^{(4)}$. Além disso, a frequente exposição à dor e ao sofrimento provoca interrupção da sua vida normal, incluindo ausências escolares e afastamento social - interferindo, assim, na qualidade de vida. ${ }^{(4)}$

Atualmente, o impacto negativo das doenças não tem sido medido apenas considerando-se como endpoints as taxas de mortalidade e as taxas de sobrevivência. Sobretudo, grande foco tem sido dado ao impacto na qualidade de vida dos pacientes, principalmente aqueles com doenças crônicas. ${ }^{(5)}$ Medidas não farmacológicas têm sido bastante estudadas, com enfoque na melhoria da qualidade de vida. Tais medidas são definidas como "um conjunto variado de sistemas, práticas e produtos médicos e de saúde que não são considerados parte integrante da medicina convencional". ${ }^{(6)}$ Dentre elas, podemos citar as 
terapias psicológicas, a exemplo da musicoterapia; as terapias físicas, como os exercícios, e as terapias psicofísicas, como a ioga. ${ }^{(6)}$

Com base nas repercussões que o câncer e seu tratamento geram à população infantojuvenil, considerase oportuno identificar e analisar as intervençóes não farmacológicas direcionadas a essa clientela, as quais poderão auxiliar os profissionais de saúde a otimizar a qualidade de vida desta população. Portanto, o objetivo deste estudo foi identificar, na literatura nacional e internacional, pesquisas sobre intervençôes não farmacológicas utilizadas para melhoria da qualidade de vida de crianças e adolescentes com câncer.

\section{Métodos}

Realizou-se uma revisão integrativa da literatura. ${ }^{(7)}$ Delimitaram-se as seguintes etapas para o desenvolvimento da pesquisa: a identificação do tema e seleção da questão de pesquisa; o estabelecimento de critérios para inclusão e exclusão de estudos; a definição das informaçóes a serem extraídas dos estudos selecionados e categorização dos estudos; a avaliação dos estudos incluídos na revisão integrativa; a interpretação dos resultados, apresentação da revisão; e a síntese do conhecimento. ${ }^{(8)}$

Determinaram-se, como tema, as intervençóes não farmacológicas utilizadas para melhoria da qualidade de vida de crianças e adolescentes com câncer, objetivando responder à seguinte questão norteadora: "Quais são as intervençôes não farmacológicas presentes na literatura nacional e internacional utilizadas para melhorar a qualidade de vida de crianças e adolescentes com câncer?”. Para a construçáo da pergunta adequada para a resolução da questão clínica pesquisada, utilizou-se a estratégia $\mathrm{PICO}^{(9)}$ - com "P" correspondendo à população (crianças e adolescentes); "I" à intervenção (intervençôes não farmacológicas); "C" à comparação (não se aplica, pois esse não é um estudo comparativo) e "O" correspondendo ao desfecho (melhoria da qualidade de vida).

Utilizaram-se, como descritores controlados identificados nos Descritores em Ciência da Saúde (DECs), Medical Subject Headings (MESH), PsycINFO Thesaurus e CINAHL Headings: "crian- ça” (“child”), "adolescente” (“adolescent"), "neoplasia" ("neoplasms") e "qualidade de vida" ("quality of life"), além das palavras-chave: "câncer" (“cancer") e “intervenção" (“intervention”). A estratégia de busca ocorreu a partir de suas diferentes combinaçóes, utilizando-se o operador booliano $A N D$ nos idiomas português e inglês, dependendo da base pesquisada.

A coleta de dados ocorreu entre os meses de setembro e outubro de 2018. As bases de dados pesquisadas foram MEDLINE ${ }^{\oplus}$ (PubMed $^{\oplus}$ ), PsycINFO, Web of Science e Cumulative Index of Nursing and Allied Health (CINAHL). Também realizou-se a pesquisa no portal da Biblioteca Virtual da Saúde, nas seguintes bases das ciências da saúde: Literatura Latino-Americana e do Caribe em Ciências da Saúde (LILACS), Índice Bibliográfico Español de Ciencias de la Salud (IBECS), MEDLINE ${ }^{\oplus}$ (BVS) e Banco de Dados em Enfermagem (BDENF).

Foram incluídos artigos originais, cuja temática respondesse à pergunta norteadora, publicados nos idiomas inglês, português ou espanhol, entre os anos 2013 e 2018. Excluíram-se estudos que focavam outras temáticas, população adulta, artigos de revisão, dissertaçóes e teses.

Foi realizada leitura exaustiva dos títulos e dos resumos, de forma independente, entre dois autores, para assegurar que os textos contemplavam a pergunta norteadora da revisão e atendiam aos critérios de inclusão estabelecidos. Em caso de dúvida a respeito da seleção, optou-se por incluir, inicialmente, a publicação e decidir sobre sua seleção somente após a leitura na íntegra de seu conteúdo.

A análise dos dados da revisão integrativa foi elaborada de forma descritiva. Utilizou-se um quadro, elaborado pelos autores, para a extração e a síntese dos dados de cada estudo primário incluído na revisão, contendo as seguintes informaçóes: título do artigo, país de origem, área de atuação dos autores, ano de publicação, objetivos, participantes, intervenções utilizadas, método de avaliação da intervenção, delineamento do estudo, nível de evidência e principais resultados e conclusões. Este quadro permitiu a comparação e a organizaçáo dos dados, de acordo as suas diferenças, as similaridades e a pergunta da revisão, os quais foram analisados criticamente e agrupados em três categorias. ${ }^{(10)}$ 
O nível de evidência foi identificado com base no delineamento do estudo. Dessa forma, atribuiuse I para revisóes sistemáticas e metanálise de ensaios clínicos randomizados; II para ensaios clínicos randomizados; III para ensaio controlado não randomizado; IV para estudos caso-controle ou coorte; $\mathrm{V}$ para revisóes sistemáticas de estudos qualitativos ou descritivos; VI para estudos qualitativos ou descritivos e VII para parecer de autoridades e/ou relatórios de comitês de especialistas. Esta hierarquia classifica os níveis I e II como fortes, III a V como moderados e VI a VII como fracos. ${ }^{(1)}$

\section{Resultados}

As buscas nas bases de dados pesquisadas capturaram 2.549 referências, sendo 1.744 na MEDLINE ${ }^{\oplus}$ $\left(\right.$ PubMed $\left.^{\oplus}\right), 150$ na CINAHL, 94 na PsycINFO, 513 na Web of Science e 48 nas demais bases (40 MEDLINE $^{\oplus}$ (BVS), 8 LILACS e nenhum nas bases IBECS e BDENF). Foram excluídos 2.508 artigos (Figura 1). Após a exclusão de 19 repetiçóes, sele- cionaram-se 22 estudos para serem lidos na íntegra, sendo todos incluídos nos resultados desta revisão.

A caracterizaçáo dos estudos selecionados, segundo variáveis de interesse estão representadas no quadro 1.

Dentre os países onde foram realizadas as pesquisas, identificou-se predomínio dos Estados Unidos, ${ }^{(16-18,26,27,30)}$ com seis trabalhos realizados, seguidos da Alemanha ${ }^{(13,14,28)}$ e do Japão, ${ }^{(19,20,22)}$ com três trabalhos cada, e Canadá(23,31) e Holanda, ${ }^{(24,25)}$ também com dois trabalhos cada. Países como China, ${ }^{(21)}$ Peru, ${ }^{(12)}$ Irã, ${ }^{(15)}$ Israel, ${ }^{(29)}$ Brasil $^{(32)}$ e Suécia ${ }^{(33)}$ aparecem com apenas um estudo cada, demonstrando ainda limitado número de pesquisas na temática.

Com relação à área de inserção dos autores, a maioria dos estudos (oito) era proveniente da área multidisciplinar (seis da medicina com psicologia, ${ }^{(18,23,24,25,26,33)}$ um da medicina e enfermagem ${ }^{(27)} \mathrm{e}$ outro que englobava três áreas: medicina, enfermagem e medicina veterinária). ${ }^{(30)}$ Outros seis estudos foram conduzidos exclusivamente por autores da

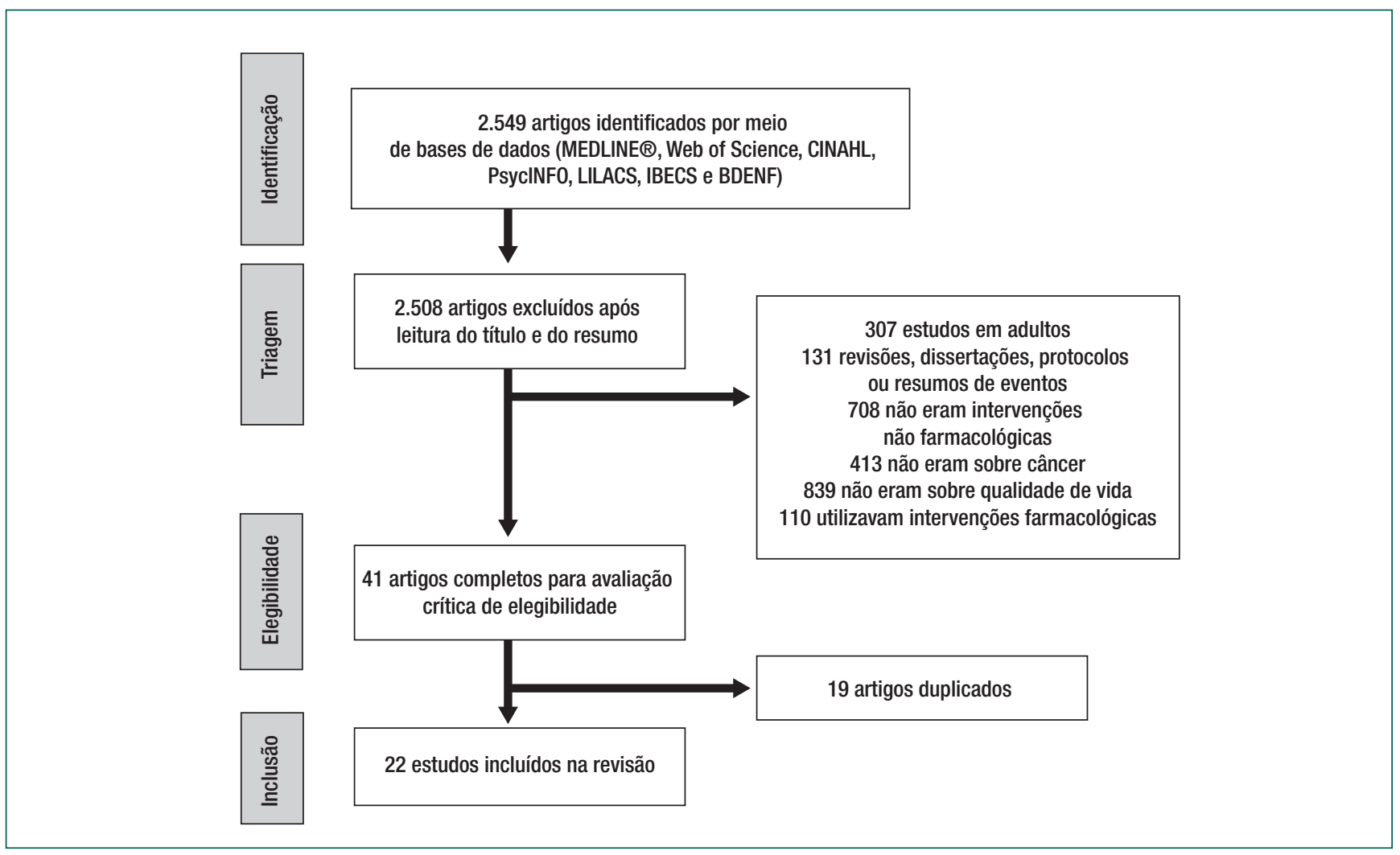

Figure 1. Fluxograma 
Quadro 1. Caracterização dos estudos selecionados, segundo variáveis de interesse.

\begin{tabular}{|c|c|c|c|c|}
\hline $\begin{array}{l}\text { Citação/Ano/Pais/ } \\
\text { Área de Atuação }\end{array}$ & Participantes & Método & Principais resultados & $\begin{array}{l}\text { Nível de } \\
\text { evidência }\end{array}$ \\
\hline \multicolumn{5}{|c|}{ Intervenções baseadas em atividade física exclusivamente } \\
\hline $\begin{array}{l}\text { Tanir; Kuguoglu } \\
2013 \\
\text { Peru } \\
\text { Enfermagem }\end{array}$ & $\begin{array}{l}40 \text { crianças com LLA } \\
\text { entre } 8 \text { e } 12 \text { anos }\end{array}$ & $\begin{array}{l}\text { Ensaio clínico randomizado } \\
\text { Grupo intervenção }(n=19) \\
\text { Intervenção: Atividade física, como caminhada e escalada. } \\
\text { Grupo controle ( } \mathrm{n}=21) \text {. } \\
\text { Instrumentos: PedsQL módulos genérico4.0 e câncer } 3.0\end{array}$ & $\begin{array}{l}\text { Escores de dor, e machucados, náusea e ansiedade relacionada ao } \\
\text { procedimento não tiveram diferenças significativas entre os grupos } \\
\text { intervenção e controle. No entanto, dentro do grupo que participou } \\
\text { da intervenção houve aumento significativo destes escores. }\end{array}$ & $\|$ \\
\hline $\begin{array}{l}\text { Beulertz et al. }{ }^{(13)} \\
2016 \\
\text { Alemanha } \\
\text { Medicina }\end{array}$ & $\begin{array}{l}33 \text { crianças com } \\
\text { câncer entre } 4 \text { e } 17 \\
\text { anos em atendimento } \\
\text { ambulatorial. }\end{array}$ & $\begin{array}{l}\text { Ensaio clínico randomizado } \\
\text { Grupo intervenção }(n=20) \\
\text { Intervenção: Atividade física por } 6 \text { meses, } 1 \text { vez por semana por } \\
60 \text { minutos. } \\
\text { Grupo controle }(n=13) \\
\text { Instrumento: Questionário KINDL }\end{array}$ & $\begin{array}{l}\text { Foram encontradas diferenças significativas no bem-estar físico } \\
\text { e emocional entre os grupos. Além disso, houve diferença } \\
\text { significativa no bem-estar emocional desde o início até a pós- } \\
\text { intervenção favorecendo aqueles que praticaram os exercícios. }\end{array}$ & $\|$ \\
\hline $\begin{array}{l}\text { Müller et al. }{ }^{(14)} \\
2016 \\
\text { Alemanha } \\
\text { Medicina }\end{array}$ & $\begin{array}{l}150 \text { crianças, } 4 \text { e } 18 \\
\text { anos, após tratamento } \\
\text { para leucemia, } \\
\text { llinfoma, tumor } \\
\text { cerebral ou sarcoma. } \\
\end{array}$ & $\begin{array}{l}\text { Ensaio controlado não randomizado (quase Experimento) } \\
\text { Intervenção: Atividade física de } 4 \text { semanas. } \\
\text { Instrumento: Questionário KINDL® }\end{array}$ & $\begin{array}{l}\text { Identificou-se efeitos imediatos e persistentes na QVRS de crianças } \\
\text { e adolescentes com diferentes tipos de câncer. No entanto, } \\
\text { pacientes com sarcoma relataram escores globais e de bem-estar } \\
\text { físico maiores, do que comparado ao pacientes com leucemia e } \\
\text { linfoma. }\end{array}$ & III \\
\hline $\begin{array}{l}\text { Khodashenas et } \\
\text { al.(15) } \\
2017 \\
\text { Irã } \\
\text { Medicina }\end{array}$ & $\begin{array}{l}20 \text { crianças com } \\
\text { câncer entre } 5 \text { e } 12 \\
\text { anos. }\end{array}$ & $\begin{array}{l}\text { Ensaio clínico randomizado } \\
\text { Grupo intervenção }(\mathrm{n}=10) \\
\text { Intervenção: Atividade física aeróbica } \\
\text { Grupo controle }(\mathrm{n}=10) \\
\text { Instrumentos: PedsQL módulo genérico } 4.0 \text { e câncer } 3.0 . \\
\end{array}$ & $\begin{array}{l}\text { Melhora no bem estar de crianças com câncer, sendo demostrado } \\
\text { melhora na sub escala dor e machucado, além de uma melhora no } \\
\text { desempenho escolar, segundo o relato dos pais. }\end{array}$ & $\|$ \\
\hline $\begin{array}{l}\text { Mendoza et al. }{ }^{(16)} \\
2017 \\
\text { Estados Unidos } \\
\text { Medicina }\end{array}$ & $\begin{array}{l}59 \text { adolescentes de } \\
14 \text { a } 18 \text { anos de } \\
\text { idade, com mais de } \\
1 \text { ano após terapia } \\
\text { oncológica. }\end{array}$ & $\begin{array}{l}\text { Ensaio clínico randomizado } \\
\text { Intervenção: Atividade física (caminhada) de } 10 \text { semanas com um } \\
\text { dispositivo de rastreamento (Fitbit Flex) e um grupo de suporte } \\
\text { virtual baseado em grupo do Facebook. } \\
\text { Instrumentos: PedsQL módulos genérico } 4.0 \text { e câncer } 3.0\end{array}$ & $\begin{array}{l}\text { Diminuição significativa no funcionamento social e ausência de } \\
\text { alterações nos outros domínios da escala. Houve boa aceitabilidade } \\
\text { e aumento da motivação para intervenção }\end{array}$ & $\|$ \\
\hline $\begin{array}{l}\text { Sparrow et al.(17) } \\
2017 \\
\text { Estados Unidos } \\
\text { Medicina }\end{array}$ & $\begin{array}{l}9 \text { crianças de } 2 \text { a } 12 \\
\text { anos, diagnosticadas } \\
\text { com tumor cerebral } \\
\text { e hemiplegia após } 0 \\
\text { término da terapia. }\end{array}$ & $\begin{array}{l}\text { Ensaio controlado não randomizado (quase Experimento) } \\
\text { Intervenção: Atividade física (terapia do movimento), com } 15 \\
\text { sessões de terapia de três horas. } \\
\text { Instrumentos: Inventário Pediátrico de Qualidade de Vida SF-15 e } \\
\text { PedsQL módulo genérico } 4.0 \text { (relato dos pais) }\end{array}$ & $\begin{array}{l}\text { Apesar de escores de QVRS maiores após a intervenção a diferença } \\
\text { não foi significativa. }\end{array}$ & III \\
\hline $\begin{array}{l}\text { Howell et al. }{ }^{(18)} \\
2018 \\
\text { Estados Unidos } \\
\text { Psicologia e } \\
\text { medicina }\end{array}$ & $\begin{array}{l}78 \text { crianças com } \\
\text { câncer entre } 11 \text { e } \\
15 \text { anos. }\end{array}$ & $\begin{array}{l}\text { Ensaio clínico randomizado } \\
\text { Grupo intervenção }(\mathrm{n}=53) \\
\text { Intervenção: Atividade física. Grupo intervenção recebeu materiais } \\
\text { educativos, um monitor de atividade e acesso a um site interativo } \\
\text { projetado para incentivar atividade física, por meio de recompensas. } \\
\text { Grupo controle }(\mathrm{n}=25) \\
\text { Instrumento: PedsQL módulo genérico } 4.0\end{array}$ & $\begin{array}{l}\text { Grupos que realizam atividade física demonstraram resultados } \\
\text { melhores de QVRS geral e na subescala de desempenho físico. }\end{array}$ & $\|$ \\
\hline \multicolumn{5}{|c|}{ Intervenções baseadas em atividades física e educativa } \\
\hline $\begin{array}{l}\text { Li et al.(19) } \\
2013 \\
\text { Japão } \\
\text { Enfermagem }\end{array}$ & $\begin{array}{l}71 \text { crianças } \\
\text { sobreviventes de } \\
\text { câncer entre } 9 \text { e } 16 \\
\text { anos. }\end{array}$ & $\begin{array}{l}\text { Ensaio clínico randomizado } \\
\text { Grupo experimental }(n=34) \\
\text { Intervenção:Programa integrado de } 4 \text { dias de treinamento baseado } \\
\text { em aventura e educação em saúde. } \\
\text { Grupo controle }(n=37) \\
\text { Instrumento: PedsQL módulo genérico } 4.0\end{array}$ & $\begin{array}{l}\text { Não houve mudança estatisticamente significativa na qualidade de } \\
\text { vida das crianças }\end{array}$ & $\|$ \\
\hline $\begin{array}{l}\text { Chung et al. }{ }^{(20)} \\
2015 \\
\text { Japão } \\
\text { Enfermagem }\end{array}$ & $\begin{array}{l}69 \text { sobreviventes, } \\
\text { com idade média de } \\
12.6 \text { anos. }\end{array}$ & $\begin{array}{l}\text { Ensaio controlado não randomizado (quase Experimento) } \\
\text { Grupo intervenção ( } \mathrm{n}=33 \text { ) } \\
\text { Intervenção: Atividade física e educação em saúde. Grupo controle } \\
\text { (n=36): Cuidados médicos regulares. } \\
\text { Instrumento: PedsQL Módulo genérico } 4.0\end{array}$ & $\begin{array}{l}\text { Melhora na QVRS, principalmente no funcionamento físico e } \\
\text { emocional. }\end{array}$ & III \\
\hline $\begin{array}{l}\text { Lam et al.(21) } \\
2018 \\
\text { China } \\
\text { Enfermagem }\end{array}$ & $\begin{array}{l}70 \text { crianças com } \\
\text { câncer entre } 9 \text { e } 18 \\
\text { anos. }\end{array}$ & $\begin{array}{l}\text { Ensaio clínico randomizado } \\
\text { Grupo intervenção }(n=37) \\
\text { Intervenção: } 15 \text { minutos educação em saúde e atividade física } \\
\text { Grupo controle ( } n=33) \\
15 \text { minutos educação em saúde e jogar cartas ou jogos de } \\
\text { xadrez e fornecer conselhos de saúde sobre prevenção da gripe e } \\
\text { importância de uma dieta saudável. } \\
\text { Instrumento: PedsQL módulo câncer 3.0. } \\
\end{array}$ & $\begin{array}{l}\text { Participantes do grupo intervenção apresentaram melhor qualidade } \\
\text { de vida do que os participantes do grupo controle nos } 9 \text { meses de } \\
\text { acompanhamento. }\end{array}$ & $\|$ \\
\hline $\begin{array}{l}\text { Li et al.(22) } \\
2018 \\
\text { Japão } \\
\text { Enfermagem }\end{array}$ & $\begin{array}{l}222 \text { crianças } \\
\text { sobreviventes de } \\
\text { câncer de } 9 \text { a } 16 \\
\text { anos. }\end{array}$ & $\begin{array}{l}\text { Ensaio clínico randomizado } \\
\text { Grupo intervenção ( } \mathrm{n}=117) \\
\text { Atividade física (baseada em aventuras), } 4 \mathrm{x} / \text { semana, } 12 \text { meses. } \\
\text { Grupo controle ( } \mathrm{n}=105) \\
\text { Atividades de lazer, } 4 \text { vezes por semana, por } 6 \text { meses. } \\
\text { Instrumento: PedsQL módulo genérico } 4.0 \text {. }\end{array}$ & $\begin{array}{l}\text { Grupo intervenção relatou melhor qualidade de vida do que o grupo } \\
\text { controle. }\end{array}$ & II \\
\hline \multicolumn{5}{|c|}{ Intervenções baseadas em atividades física e psicológica } \\
\hline $\begin{array}{l}\text { Wurz et al.(23) } \\
2014 \\
\text { Canadá } \\
\text { Psicologia e } \\
\text { medicina }\end{array}$ & $\begin{array}{l}8 \text { crianças com } \\
\text { câncer entre } 5 \text { e } 17 \\
\text { anos. }\end{array}$ & $\begin{array}{l}\text { Ensaio controlado não randomizado (quase experimento) } \\
\text { Intervenção: Sessões supervisionadas de Yoga } 2 \text { vezes / semana. } \\
\text { por } 12 \text { semanas. } \\
\text { Instrumento: PedsQL modulo genérico 4.0. }\end{array}$ & $\begin{array}{l}\text { Melhorias significativas no escore total de QVRS e na subescala: } \\
\text { psicossocial,. além de melhora no funcionamento físico, e escolar, } \\
\text { segundo relato dos pais. }\end{array}$ & III \\
\hline
\end{tabular}




\begin{tabular}{|c|c|c|c|c|}
\hline $\begin{array}{l}\text { Citação/Ano/Pais/ } \\
\text { Área de Atuação }\end{array}$ & Participantes & Método & Principais resultados & \begin{tabular}{|l|} 
Nível de \\
evidência
\end{tabular} \\
\hline $\begin{array}{l}\text { Van Dijk-Lokkart } \\
\text { et al. }{ }^{24)} \\
2015 \\
\text { Holanda } \\
\text { Psicologia e } \\
\text { medicina }\end{array}$ & $\begin{array}{l}68 \text { crianças com } \\
\text { câncer entre } 8 \text { e } 18 \\
\text { anos. }\end{array}$ & $\begin{array}{l}\text { Ensaio clínico randomizado } \\
\text { Grupo intervenção ( } n=30 \text { ) / Intervenção: Exercício físico (45 min } \\
\text { por } 12 \text { semanas) mais intervenção psicossocial. } \\
\text { Grupo controle ( } n=38 \text { ) / Instrumentos: PedsQL módulos genérico } \\
4.0 \text { e câncer } 3.0\end{array}$ & $\begin{array}{l}\text { Nenhum efeito sobre a QVRS foi identificado no auto relato } \\
\text { da criança. No entanto, no relato dos pais, notou-se melhora } \\
\text { significativa nos níveis da sub escala dor, menos ansiedade em } \\
\text { curto prazo e menos náuseas em longo prazo. }\end{array}$ & $\|$ \\
\hline $\begin{array}{l}\text { Van Dijk-Lokkart } \\
\text { et al. } 25 \text { ) } \\
2015 \text { / Holanda } \\
\text { Psicologia e } \\
\text { medicina }\end{array}$ & $\begin{array}{l}61 \text { crianças com } \\
\text { câncer, entre } 8 \text { e } 18 \\
\text { anos. }\end{array}$ & \begin{tabular}{|l|} 
Ensaio clínico randomizado \\
Intervenção: Programa de intervenção física e psicossocial \\
Instrumento: PedsQL módulos genérico 4.0 e câncer 3.0
\end{tabular} & $\begin{array}{l}\text { Os resultados não demonstraram eficácia da intervenção na QVRS } \\
\text { de crianças e adolescentes com câncer }\end{array}$ & $\|$ \\
\hline $\begin{array}{l}\text { Orsey et al.(26) } \\
2017 \\
\text { Estados Unidos } \\
\text { Psicologia e } \\
\text { medicina }\end{array}$ & $\begin{array}{l}20 \text { crianças e } \\
\text { adolescentes } \\
\text { em tratamento } \\
\text { oncológico, } 8 \text { e } 18 \\
\text { anos, e seus pais. }\end{array}$ & \begin{tabular}{|l} 
Ensaio controlado não randomizado (quase Experimento) \\
Intervenção: Yoga por 8 semanas \\
Instrumentos: PedsQL módulos genérico 4.0 e câncer 3.0.
\end{tabular} & $\begin{array}{l}\text { Identificou aumento significativo da função emocional, social e total } \\
\text { da QVRS. }\end{array}$ & III \\
\hline \multicolumn{5}{|c|}{ Intervenções psicológicas exclusivamente } \\
\hline $\begin{array}{l}\text { Akard et al. }{ }^{(27)} \\
2015 \\
\text { Estados Unidos } \\
\text { Enfermagem e } \\
\text { medicina }\end{array}$ & $\begin{array}{l}28 \text { crianças com } \\
\text { câncer de } 7 \text { a } 17 \\
\text { anos. }\end{array}$ & $\begin{array}{l}\text { Ensaio clínico randomizado } \\
\text { Grupo de intervenção }(n=15) \\
\text { Intervenção: Legacy-Making. Entrevistas individuais com crianças } \\
\text { com câncer avançado sobre o que gostariam que sua família e } \\
\text { amigos lembrassem sobre elas e escreverem palavras ou fizeram } \\
\text { algo especial para dar a alguém significativo para elas. } \\
\text { Grupo controle ( } n=13) \text {. } \\
\text { Instrumento: PedsQL módulo genérico 4.0. }\end{array}$ & \begin{tabular}{|l|} 
Não houve diferença significativa nos escores de QVRS embora os \\
pais percebam que a intervenção facilitou a comunicação entre pais \\
e filhos, foi uma estratégia de enfrentamento e ajudou as crianças a \\
lidar com a doença, se sentir melhor emocionalmente, socialmente, \\
fisicamente e espiritualmente.
\end{tabular} & $\|$ \\
\hline $\begin{array}{l}\text { Malboeuf-Hurtubise } \\
\text { et al.(28) } \\
2016 \\
\text { Alemanha } \\
\text { Psicologia }\end{array}$ & $\begin{array}{l}14 \text { adolescentes com } \\
\text { câncer entre } 11 \text { e } \\
18 \text { anos. }\end{array}$ & $\begin{array}{l}\text { Ensaio controlado não randomizado (quase experimento) } \\
\text { Intervenção: Mindfulness } \\
\text { Instrumento: PedsQL módulo câncer } 3.0\end{array}$ & $\begin{array}{l}\text { Todos os participantes relataram gostar do grupo. Houve diminuição } \\
\text { do estresse antes dos tratamentos, facilidade para dormir mais } \\
\text { rápido e nenhum relato de tristeza ou depressão No entanto, } \\
\text { as diferenças entre grupos pré e pós intervenção não foram } \\
\text { significativas para a QV. }\end{array}$ & III \\
\hline $\begin{array}{l}\text { Shoshani et al. }{ }^{(29)} \\
2016 \\
\text { Israel } \\
\text { Psicologia }\end{array}$ & $\begin{array}{l}66 \text { crianças com } \\
\text { câncer com risco } \\
\text { de vida entre } 5 \text { e } 12 \\
\text { anos. }\end{array}$ & \begin{tabular}{|l} 
Ensaio clínico randomizado \\
Grupo intervenção ( $n=32)$ \\
Intervençã̃o: Make a wish. Evocar esperança e alegria para o \\
enfrentamento da doença, satisfazendo o maior desejo da criança. \\
Grupo de controle $(n=34)$ / Instrumento: PedsQL 4.0. \\
\end{tabular} & $\begin{array}{l}\text { Crianças do grupo de intervenção exibiram uma redução } \\
\text { significativa no sofrimento geral, depressão e sintomas de } \\
\text { ansiedade, melhor qualidade de vida relacionada à saúde, } \\
\text { esperança e afeto positivo. }\end{array}$ & $\|$ \\
\hline $\begin{array}{l}\text { McCullouh et al. }{ }^{(30)} \\
2017 \\
\text { Estados Unidos } \\
\text { Enfermagem, } \\
\text { medicina e } \\
\text { veterinária } \\
\end{array}$ & $\begin{array}{l}106 \text { crianças recém } \\
\text { diagnosticadas com } \\
\text { câncer entre } 3 \text { e } 17 \\
\text { anos. }\end{array}$ & \begin{tabular}{|l|} 
Ensaio clínico randomizado \\
Grupo intervenção ( $\mathrm{n}=60)$ \\
Intervenção: Terapia Assistida por animais \\
Grupo controle $(n=46)$ \\
Instrumentos: PedsQL módulos genérico 4.0 e câncer 3.0.
\end{tabular} & $\begin{array}{l}\text { Não houve diferenças significativas entre os grupos ao longo do } \\
\text { tempo. }\end{array}$ & $\|$ \\
\hline $\begin{array}{l}\text { Barrera et al. }{ }^{(31)} \\
2018 \\
\text { Canadá } \\
\text { Psicologia }\end{array}$ & $\begin{array}{l}91 \text { crianças com } \\
\text { tumor cerebral/ } \\
\text { espinhal entre } 8 \text { e } \\
16 \text { anos. }\end{array}$ & $\begin{array}{l}\text { Ensaio clínico randomizado } \\
\text { Grupo intervenção }(n=43) \\
\text { Intervenção: Habilidades sociais (amizade; cooperação; } \\
\text { gerenciando provocações e bullying; resolução de conflitos; } \\
\text { empatia; e afirmaçã). } \\
\text { Grupo controle }(\mathrm{n}=48) \text { / Instrumento: PedsQL mód. genérico 4.0. }\end{array}$ & Não houve diferença significativa na qualidade de vida. & $\|$ \\
\hline $\begin{array}{l}\text { Silva; Osório(32) } \\
2018 \\
\text { Brasil } \\
\text { Medicina }\end{array}$ & $\begin{array}{l}24 \text { crianças, } 6 \text { e } 12 \\
\text { anos, com tumor } \\
\text { sólido ou LLA. }\end{array}$ & $\begin{array}{l}\text { Ensaio controlado não randomizado (quase experimento) } \\
\text { Intervenção: Terapia Assistida por Animais } \\
\text { Instrumento: Escala de Avaliação da Qualidade de Vida. }\end{array}$ & Não houve diferença significativa nos escores de QV. & III \\
\hline $\begin{array}{l}\text { Uggla et al.(33) } \\
2018 \\
\text { Suécia } \\
\text { Psicologia e } \\
\text { medicina }\end{array}$ & $\begin{array}{l}29 \text { crianças, } 0 \text { e } 17 \\
\text { anos, submetidas } \\
\text { ao Transplante } \\
\text { de Células Tronco } \\
\text { Hematopoiéticas. }\end{array}$ & \begin{tabular}{|l} 
Ensaio clínico randomizado \\
Grupo intervenção $(n=14)$ \\
Intervenção: Musicoterapia \\
Grupo controle ( $(n=15)$. \\
Instrumentos: PedsQL módulos genérico 4.0 e câncer 3.0.
\end{tabular} & $\begin{array}{l}\text { Melhora significativa em todos os domínios da escala genérica, } \\
\text { exceto o escolar emelhora significativas nas subescalas ansiedade } \\
\text { relacionada ao tratamento, preocupações, problemas cognitivos e } \\
\text { comunicação no módulo câncer. }\end{array}$ & II \\
\hline
\end{tabular}

medicina, ${ }^{(13-17,32)}$ seguidos por cinco estudos da enfermagem $^{(12,19-22)}$ e três da psicologia. ${ }^{(28,29,31)}$

Os instrumentos para avaliar a qualidade de vida foram o Pediatric Quality of Life Inventory ${ }^{\mathrm{TM}}\left(\mathrm{Peds}_{\mathrm{Q}} \mathrm{L}^{\mathrm{TM}}\right)$ - módulos genérico 4.0 $0^{(12,15-20,22-27,29,30-33)}$ e de câncer 3.0; ${ }^{(12,16,21,24-26,30,32)}$ questionário $\mathrm{KINDL}^{\oplus(13,14)}$ e Escala de Avaliação da Qualidade de Vida. ${ }^{(32)}$ Salienta-se que alguns estudos utilizaram mais de um instrumento para avaliar a qualidade de vida.
Já, com relação ao delineamento dos estudos, 15 eram ensaios clínicos randomizados, ${ }^{(12,13,15,16,18,19,21,22,24,25,27,29-31,33)}$ sendo classificados como nível de evidência II, e sete ensaios controlados não randomizados (quase experimento), com nível de evidência III. ${ }^{(14,17,20,23,28,26,32)}$

Dos 22 estudos, sete utilizaram atividade física exclusiva, ${ }^{(12-18)}$ sendo cinco com resultados significativos na melhoria da qualidade de vida. ${ }^{(12-15,18)}$ Oito 
realizaram atividade física conciliada a outra intervenção ${ }^{(19-26)}$, sendo quatro conciliados com educação em saúde ${ }^{(19-22)}$ e quatro com atividade psicológica $^{(23-26)}$ (sendo seis significativos $\left.{ }^{(20-24,26)}\right)$. Sete estudos aplicaram intervençóes psicológicas, sendo duas terapias com animais; ${ }^{30,32)}$ uma musicoterapia; ${ }^{(33)}$ uma prática de atenção plena (mindfulness); ${ }^{(28)}$ uma intervençáo baseada no desenvolvimento de habilidades sociais; ${ }^{(31)}$ uma atividade envolvendo desejos $^{(29)}$ e outra atividade o legado que as crianças gostariam de deixar $^{(27)}$ (dois significativos ${ }^{(29,33)}$ ).

Os estudos foram agrupados empiricamente, com base nas intervenções não farmacológicas realizadas para melhorar a qualidade de vida de crianças e adolescentes com câncer, sendo definidas três categorias: intervenções físicas; intervençôes físicas conciliadas a ações educativas ou psicológicas; e intervenções psicológicas.

\section{Categoria 1. Intervenções físicas}

Sete estudos utilizaram exercícios físicos exclusivamente como intervenção ${ }^{(12-18)}$ e cinco deles $(71,4 \%)$ demonstraram melhora significativa da qualidade de vida. ${ }^{(12-15,18)}$

O primeiro estudo da revisão utilizou a caminhada como atividade física. Nele, 59 adolescentes, de 14 a 18 anos, sobreviventes do câncer, tiveram seus passos contabilizados por um dispositivo de rastreamento (Fitbit Flex ${ }^{\mathrm{TM}}$ ). Os resultados demonstraram melhor qualidade de vida relacionada à saúde no funcionamento social da PedsQL $\mathrm{L}^{\mathrm{m}}$ - módulo genérico do grupo controle. Os outros domínios da escala não apresentaram alteraçôes significativas, mesmo tendo boa aceitabilidade e demonstrando aumento da motivação. ${ }^{(16)}$

No segundo estudo, utilizaram-se exercícios de reabilitação simples por brincadeiras e práticas de vida diária com crianças de 2 a 12 anos com tumor cerebral e hemiplegia. Nove pais participaram do estudo e relataram que os escores de qualidade de vida, relacionada à saúde da criança, melhoraram ou permaneceram estáveis durante o período do estudo, sem demonstrar diferença significativa. ${ }^{(17)}$

O terceiro estudo utilizou exercícios aeróbicos (caminhada, corrida e jogos) com 20 crianças com câncer de 5 a 12 anos. $\mathrm{O}$ exercício programado de
12 semanas foi capaz de melhorar o bem-estar de crianças, sendo demostrado aumento da qualidade de vida relacionada à saúde na subescala dor e machucados do PedsQL ${ }^{m}$ - módulo câncer, além de melhora no funcionamento escolar na PedsQL ${ }^{m}-$ módulo genérico, segundo o relato dos pais. ${ }^{(15)}$

Outro estudo verificou a eficácia de exercícios (corrida, salto à distância e salto lateral) em 33 crianças com câncer, de 4 a 17 anos, em atendimento ambulatorial. Identificaram-se diferenças significativas na qualidade de vida, no bem-estar físico e emocional entre os grupos. Além disso, foi detectada diferença significativa para o bem-estar emocional desde o início até a pós-intervenção, favorecendo aqueles que praticaram exercícios. ${ }^{(13)}$

Em investigação conduzida com 40 crianças, de 8 a 12 anos, com diagnóstico de leucemia linfoide aguda, os pacientes participaram de um programa de exercícios de 3 meses (amplitude de movimento ativa, fortalecimento muscular da perna e exercícios aeróbicos). Os resultados demonstraram que os escores de dor e machucados, náusea e ansiedade relacionados ao procedimento não tiveram diferenças significativas entre os grupos intervenção e controle. No entanto, dentro do mesmo grupo que participou da intervenção, houve aumento significativo. ${ }^{(12)}$

O sexto estudo realizou um programa de reabilitação com 150 crianças, de 4 a 18 anos, após o término do tratamento para leucemia, linfoma, tumor cerebral ou sarcoma. Observaram-se efeitos imediatos e persistentes na qualidade de vida relacionada à saúde de crianças e adolescentes nos diferentes tipos de câncer. No entanto, pacientes com sarcoma relataram escores globais e de bem-estar físico maiores quando comparados aos pacientes com leucemia e linfoma. ${ }^{(14)}$

O sétimo estudo avaliou o estímulo à atividade física, por meio de recompensa, em 78 crianças de 11 a 15 anos, sobreviventes de câncer, havendo melhora na qualidade de vida relacionada à saúde no escore geral e na subescala de desempenho físico. ${ }^{(18)}$

\section{Categoria 2. Intervenções físicas conciliadas a ações educativas ou psicológicas}

Oito estudos compuseram essa categoria, ${ }^{(19-26)}$ sendo que $75 \%$ deles demonstraram melhora na qualidade de vida. ${ }^{(20-24,26)}$ 
Quatro investigaçóes utilizaram exercícios físicos e ação educativa. ${ }^{(19-22)}$ Destes, dois realizaram exercícios físicos baseados em atividades de aventura, em conjunto com atividades educativas, e identificaram melhora da qualidade de vida relacionada à saúde no grupo que participou da intervenção quando comparado ao controle. Um deles tinha 69 sobreviventes (média de idade 12 anos), ${ }^{(20)}$ enquanto o outro contava com 222 crianças de 9 a 16 anos. ${ }^{\text {(22) }}$ No entanto, em outro estudo bastante semelhante, realizado com 71 crianças sobreviventes de câncer, de 9 a 16 anos, que desenvolveu educação em saúde aliada ao treinamento baseado em aventura (escalada, minijogos olímpicos e corrida), não houve efeito estatisticamente significativo na qualidade de vida das crianças. ${ }^{(19)}$

No quarto estudo, 70 crianças e adolescentes com câncer em tratamento tiveram uma palestra sobre a importância de exercícios físicos; depois, o grupo experimental passou por treinamento físico com coaching. Os participantes do grupo experimental relataram níveis significativamente melhores de qualidade de vida do que os participantes do grupo controle nos 9 meses de acompanhamento. ${ }^{(21)}$

Os outros quatro estudos desta categoria utilizaram atividades físicas aliadas a atividades psicológicas. ${ }^{(23-26)}$ Dois combinaram exercícios físicos (duas vezes por semana em um centro de fisioterapia) a um treinamento psicossocial (seis sessões, uma vez a cada 6 semanas) em mais de 60 crianças com câncer, de 8 a 18 anos; porém, um deles não mostrou eficácia na qualidade de vida, ${ }^{(25)}$ enquanto o outro identificou melhora significativa nos níveis da subescala dor, ansiedade em curto prazo e náuseas em longo prazo - a partir do relato dos pais. ${ }^{(24)}$

Outros dois estudos utilizaram a prática de ioga. Um deles, desenvolvido com crianças e adolescentes de 8 a 18 anos, mostrou aumento significativo da função emocional, social e total da escala de qualidade de vida relacionada à saúde genérica, no relato das crianças. ${ }^{(26)}$ No outro estudo, realizado com pacientes de 5 e 17 anos, os resultados mostraram melhorias significativas no escore total e psicossocial de qualidade de vida relacionada à saúde, além de melhoria no funcionamento físico e escolar, segundo o relato dos pais. ${ }^{(23)}$

\section{Categoria 3. Intervenções psicológicas}

Sete estudos utilizaram intervençóes psicológicas, ${ }^{(27-33)}$ sendo que apenas dois deles (28,6\%) foram eficazes. ${ }^{(29,33)}$

O primeiro utilizou práticas de atenção plena (mindfulness) adaptadas para 14 adolescentes, de 11 a 18 anos, com diagnóstico de câncer. Todos os participantes relataram gostar do grupo e da oportunidade de falar abertamente sobre suas emoçóes. Dentre os pontos positivos, foram citados a diminuição do estresse antes dos tratamentos, a facilidade para dormir e a ausência de tristeza ou depressão após as conversas sobre a experiência de câncer. No entanto, quantitativamente, as diferenças entre grupos pré e pós-intervenção não foram significativas para qualidade de vida, humor e sono. ${ }^{(28)}$

O segundo estudo utilizou, como intervenção, o estímulo à prática e ao desenvolvimento de habilidades sociais (amizade, cooperação, gerenciamento de provocações ou bullying, resolução de conflitos, empatia e afirmaçôes) com 91 crianças, de 8 a 16 anos, diagnosticadas com tumor cerebral. Os resultados não demonstraram diferença significativa na qualidade de vida, nem em relação ao relato dos cuidadores, nem dos professores. ${ }^{(31)}$

Em uma atividade sobre o legado, 28 crianças e adolescentes com câncer no fim da vida relataram o que gostariam que sua família e amigos lembrassem sobre eles, e escreverem palavras ou fizeram algo especial para alguém. Embora a percepção dos pais fosse a de que a intervenção facilitou a comunicação entre pais e filhos, foi uma estratégia de enfrentamento e ajudou as crianças a lidarem com a doença e a se sentirem melhor emocionalmente, socialmente, fisicamente e espiritualmente, não resultando, contudo, em diferença significativa nos escores de qualidade de vida relacionada à saúde. ${ }^{(27)}$

No quarto e quinto estudos, utilizou-se a terapia assistida por animais como intervenção em 24 crianças, de 6 a 12 anos, ${ }^{(32)}$ e em 106 crianças, de 3 a 17 anos. ${ }^{(30)}$ Apesar de encontrar melhora significativa nos níveis de dor, irritação, estresse, sintomas depressivos e ansiedade, nenhum dos estudos identificou diferenças significativas na qualidade de vida relacionada à saúde. ${ }^{(24,32)}$

O sexto estudo utilizou a musicoterapia com crianças de 0 e 17 anos durante e após o transplan- 
te de células-tronco hematopoiéticas. Os resultados demonstraram maior pontuação total na qualidade de vida genérica e melhora significativa em todos os domínios da escala, exceto no referente ao escolar. No modulo câncer, os escores foram maiores para o grupo intervenção, com melhora mais significativa para os itens de ansiedade relacionada ao tratamento, preocupaçóes, problemas cognitivos e comunicação. No relato dos pais, os resultados foram parecidos, com melhoria do funcionamento em três dos quatro domínios do módulo genérico e todos os itens do módulo câncer, indicando melhor qualidade de vida para crianças que receberam musicoterapia. ${ }^{(33)}$

No último estudo, foi feita a atividade Make-a$W_{i s h}{ }^{\oplus}$, que faz parte de uma fundação internacional que concede desejo a crianças, de 5 a 12 anos, com condiçôes de saúde graves. O objetivo desta intervenção é realizar o maior desejo da criança, acreditando que esperança e alegria potencialmente darão às crianças mais força para lidar com o câncer. Os resultados demonstraram redução significativa no sofrimento geral, depressão e sintomas de ansiedade e melhora na qualidade de vida relacionada à saúde, esperança e afeto positivo. ${ }^{(29)}$

\section{Discussão}

Os resultados indicaram crescente interesse de pesquisa na aplicação de estratégias não farmacológicas de gerenciamento de sintomas e melhoria da qualidade de vida. A maior parte dos estudos utilizou o PedsQL ${ }^{m}$ para avaliação da qualidade de vida. A eleição desse instrumento pode ter sido favorecida pelo fato de ser traduzido e validado para diversos idiomas. $^{(34)}$

Dentre as áreas de atuação dos autores, a enfermagem aparece em terceiro lugar. Considerando-se todas as repercussões do câncer na vida da criança, são imprescindíveis a sensibilização e a qualificação dos profissionais de enfermagem para o uso de intervenções voltadas para o manejo dos sintomas e a melhoria da qualidade de vida. ${ }^{(2)}$ Salienta-se a necessidade de mais estudos na área, para fornecer subsídios para qualificar o cuidado.
A maioria das pesquisas utilizou exercícios físicos, exclusivamente ou conciliados a outras estratégias, como intervençóes não farmacológicas para melhora da qualidade de vida. É compreensível que as intervençôes com o uso de exercícios estejam entre as mais exitosas. $\mathrm{O}$ uso do treinamento físico tem sido extensivamente estudado, em cenários de câncer, como parte das estratégias de prevenção ou reabilitação. ${ }^{(35)} \mathrm{O}$ exercício físico aumenta os níveis da proteína Fator Neurotrófico Derivado do Cérebro (BDNF), que está associada à melhora cognitiva e ao alívio da depressão e da ansiedade. ${ }^{(36)}$ As mudanças moleculares e sistêmicas na infiltração de células imunes e citocinas inflamatórias geradas pelo exercício podem afetar diretamente os desfechos específicos do tumor, controlando sua iniciação e progressão pela regulação das funçôes imune e inflamatória, podendo, o treinamento físico, ser utilizado como tratamento antineoplásico - se incorporado à terapia oncológica padrão em benefício dos pacientes com câncer. ${ }^{(37,38)}$ Para além destes achados, evidências emergentes sugerem que o treinamento físico também reduz o risco de 17 dentre 27 tipos de câncer. ${ }^{(39)}$

Os exercícios conciliados à ação educativa ou psicológica foram as estratégias que tiveram maior número de estudos com melhora significativa na qualidade de vida. A integração da atividade física com a educação em saúde potencializa o efeito positivo dos exercícios, pois essa uniáo promove a conscientização sobre a importância da atividade física para os participantes, corrige falsas concepçôes, aumenta a autoeficácia e promove melhor adaptação à nova vida. ${ }^{(19)}$ Outras revisóes têm mostrado que as intervenções, tanto físicas quanto psicológicas, têm auxiliado na melhora de sintomas (fadiga e estresse) e na qualidade de vida de crianças e adolescentes com câncer. ${ }^{(40-42)}$ Intervençôes psicológicas conciliadas aos exercícios físicos também podem favorecer o bem-estar e a prática de atividades físicas, melhorando a qualidade de vida dos pacientes; porém mais estudos são necessários.

Apesar de expressivo número de estudos apresentar bom nível de evidência, reconhecem-se as limitaçôes desta revisão. Os seus resultados devem ser avaliados com cautela, pois o risco de viés, de to- 
dos os estudos revisados, é relativamente alto, considerando a diversidade dos tipos de intervençóes e dos protocolos, o número de participantes bastante variado e a inexistência do uso de grupo controle e randomização em grande parte dos estudos, o que dificulta a generalização dos achados.

\section{Conclusão}

Intervenções utilizando exclusivamente exercícios físicos, ou conciliados a outra intervenção, compuseram a maioria dos estudos revisados - e estes também foram os que se mostraram mais eficazes na melhoria da qualidade de vida de crianças e adolescentes com câncer. Encontraram-se também estudos que utilizaram intervençôes psicológicas. Dentre as implicaçôes para a prática, as evidências apresentadas sensibilizam os profissionais sobre a importância da temática e enfatizam a importância do uso de intervençóes não farmacológicas em conjunto com o tratamento padrão dos pacientes. Estas intervençóes podem ser úteis no apoio às práticas clínicas, com vistas à qualificação do cuidado no planejamento de açóes estratégicas, no âmbito da enfermagem pediátrica, e, consequentemente, na melhoraria da qualidade de vida de crianças e adolescentes com câncer.

\section{Referências}

1. Brasil. Ministério da Saúde. Instituto Nacional de Câncer José Alencar Gomes da Silva (INCA). Estimativa 2018. Incidência de câncer no Brasil. Rio de Janeiro: INCA; 2018.

2. Souza ML, Reichert AP, Sá LD, Assolini FE, Collet N. Stepping into a new world: the meaning of sicken for the child with cancer. Texto Contexto Enferm. 2014;23(2):391-9.

3. Hosoda T. The impact of childhood cancer on family functioning: $A$ review. Grad Stud J Psychol. 2014;15:18-30.

4. Silva LF, Cabral IE. Cancer repercussions on play in children: implications for nursing care. Texto Contexto Enferm. 2014;23(4):935-43.

5. Tóthová V, Bártlová S, Dolák F, Kaas J, Kimmer D, Maňhalová J, et al. Quality of life in patients with chronic diseases. Neuro Endocrinol Lett. 2014;35 Suppl 1:11-8.

6. Park J, Hirz CE, Manotas K, Hooyman N. Nonpharmacological pain management by ethnically diverse older adults with chronic pain: barriers and facilitators. J Gerontol Soc Work. 2013;56(6):487-508.

7. Whittemore R, Knafl K. The integrative review: updated methodology. J Adv Nurs. 2005;52(5):546-53.
8. Mendes KD, Silveira RC, Galvão CM. Revisão integrativa: método de pesquisa para a incorporação de evidências na saúde e na enfermagem. Texto Contexto Enferm. 2008;17(4):758-64.

9. da Costa Santos CM, de Mattos Pimenta CA, Nobre MR. The PICO strategy for the research question construction and evidence search. Rev Lat Am Enfermagem. 2007;15(3):508-11.

10. Cooper H. Scientific Guidelines for Conducting Integrative Research Reviews. Rev Educ Res. 1982;52(2):291-302.

11. Melnyk BM, Fineout-Overholt E. Evidence-based practice in nursing \& healthcare. A guide to best practice. China: Wolters Kluwer/Lippincott Williams \& Wilkins; 2011.

12. Tanir MK, Kuguoglu S. Impact of exercise on lower activity levels in children with acute lymphoblastic leukemia: a randomized controlled trial from Turkey. Rehabil Nurs. 2013;38(1):48-59.

13. Beulertz J, Prokop A, Rustler V, Bloch W, Felsch M, Baumann FT. Effects of a 6-Month, Group-Based, Therapeutic Exercise Program for Childhood Cancer Outpatients on Motor Performance, Level of Activity, and Quality of Life. Pediatr Blood Cancer. 2016;63(1):127-32.

14. Müller C, Krauth KA, Gerß J, Rosenbaum D. Physical activity and health-related quality of life in pediatric cancer patients following a 4-week inpatient rehabilitation program. Support Care Cancer. 2016;24(9):3793-802.

15. Khodashenas E, Badiee Z, Sohrabi M, Ghassemi A, Hosseinzade V. The effect of an aerobic exercise program on the quality of life in children with cancer. Turk J Pediatr. 2017;59(6):678-83.

16. Mendoza JA, Baker KS, Moreno MA, Whitlock K, Abbey-Lambertz M, Waite $A$, et al. A Fitbit and Facebook mHealth intervention for promoting physical activity among adolescent and young adult childhood cancer survivors: A pilot study. Pediatr Blood Cancer. 2017;64(12):e26660.

17. Sparrow J, Zhu L, Gajjar A, Mandrell BN, Ness KK. Constraint-Induced Movement Therapy for Children With Brain Tumors. Pediatr Phys Ther. 2017;29(1):55-61.

18. Howell CR, Krull KR, Partin RE, Kadan-Lottick NS, Robison LL, Hudson $\mathrm{MM}$, et al. Randomized web-based physical activity intervention in adolescent survivors of childhood cancer. Pediatr Blood Cancer. 2018;65(8):e27216.

19. Li HC, Chung OK, Ho KY, Chiu SY, Lopez V. Effectiveness of an integrated adventure-based training and health education program in promoting regular physical activity among childhood cancer survivors. Psychooncology. 2013;22(11):2601-10.

20. Chung OK, Li HC, Chiu SY, Ho KY, Lopez V. Sustainability of an Integrated Adventure-Based Training and Health Education Program to Enhance Quality of Life Among Chinese Childhood Cancer Survivors: A Randomized Controlled Trial. Cancer Nurs. 2015;38(5):366-74.

21. Lam KK, Li WH, Chung OK, Ho KY, Chiu SY, Lam HS, et al. An integrated experiential training programme with coaching to promote physical activity, and reduce fatigue among children with cancer: A randomised controlled trial. Patient Educ Couns. 2018;101(11):1947-56.

22. Li WH, Ho KY, Lam KK, Lam HS, Chui SY, Chan GC, et al. Adventurebased training to promote physical activity and reduce fatigue among childhood cancer survivors: A randomized controlled trial. Int J Nurs Stud. 2018;83:65-74.

23. Wurz A, Chamorro-Vina C, Guilcher GM, Schulte F, Culos-Reed SN. The feasibility and benefits of a 12-week yoga intervention for pediatric cancer out-patients. Pediatr Blood Cancer. 2014;61(10):1828-34.

24. van Dijk-Lokkart EM, Braam Kl, Broeder EV, Kaspers GJ, Takken T, Grootenhuis MA, et al. Effects of a combined physical and psychosocial 
intervention program for childhood cancer patients on quality of life and psychosocial functioning: results of the QLIM randomized clinical trial. Psychooncology. 2015; 25(7):815-22.

25. van Dijk-Lokkart EM, Braam Kl, Huisman J, Kaspers GJ, Takken T, Veening MA, et al. Factors influencing childhood cancer patients to participate in a combined physical and psychosocial intervention program: Quality of Life in Motion. Psychooncology. 2015;24(4):46571.

26. Orsey $A D$, Park CL, Pulaski R, Shankar NL, Popp JM, Wakefield D. Results of a Pilot Yoga Intervention to Improve Pediatric Cancer Patients' Quality of Life and Physical Activity and Parents' Well-being. Rehabil Oncol. 2017;35:15-23.

27. Akard TF, Dietrich MS, Friedman DL, Hinds PS, Given B, Wray S, et al. Digital storytelling: an innovative legacy-making intervention for children with cancer. Pediatr Blood Cancer. 2015;62(4):658-65.

28. Malboeuf-Hurtubise C, Achille M, Muise L, Beauregard-Lacroix R, Vadnais M, Lacourse E. A mindfulness-based meditation pilot study: lessons learned on acceptability and feasibility in adolescents with cancer. J Child Fam Stud. 2016;25(4):1168-77.

29. Shoshani A, Mifano K, Czamanski-Cohen J. The effects of the Make a Wish intervention on psychiatric symptoms and health-related quality of life of children with cancer: a randomised controlled trial. Qual Life Res. 2016;25(5):1209-18.

30. McCullough A, Ruehrdanz A, Jenkins MA, Gilmer MJ, Olson J, Pawar $A$, et al. Measuring the Effects of an Animal-Assisted Intervention for Pediatric Oncology Patients and Their Parents: A Multisite Randomized Controlled Trial [Formula: see text]. J Pediatr Oncol Nurs. 2018;35(3):159-77.

31. Barrera M, Atenafu EG, Sung L, Bartels U, Schulte F, Chung J, et al. A randomized control intervention trial to improve social skills and quality of life in pediatric brain tumor survivors. Psychooncology. 2018;27(1):91-8.

32. Silva NB, Osório FL. Impact of an animal-assisted therapy programme on physiological and psychosocial variables of paediatric oncology patients. PLoS One. 2018;13(4):e0194731.
33. Uggla L, Bonde LO, Hammar U, Wrangsjö B, Gustafsson B. Music therapy supported the health-related quality of life for children undergoing haematopoietic stem cell transplants. Acta Paediatr. 2018;107(11):1986-94.

34. Varni JW. The PedsQLTM . Measurement Model for the Pediatric Quality of Life Inventory ${ }^{\text {TM }}$ [Internet]. 2019 [cited 2019 Jan 26]. Available from: http://www.pedsql.org/

35. Jones LW. Precision oncology framework for investigation of exercise as treatment for cancer. J Clin Oncol. 2015;33(35):4134-7.

36. Sleiman SF, Henry J, Al-Haddad R, El Hayek L, Abou Haidar E, Stringer $\mathrm{T}$, et al. Exercise promotes the expression of brain derived neurotrophic factor (BDNF) through the action of the ketone body $\otimes$-hydroxybutyrate. eLife. 2016;5:e15092.

37. Filler K, Lyon D, McCain N, Bennett J Jr, Fernández-Martínez JL, deAndrés-Galiana EJ, et al. Relationship of Mitochondrial Enzymes to Fatigue Intensity in Men With Prostate Cancer Receiving External Beam Radiation Therapy. Biol Res Nurs. 2016;18(3):274-80.

38. Hojman P. Exercise protects from cancer through regulation of immune function and inflammation. Biochem Soc Trans. 2017;45(4):905-11.

39. Moore SC, Lee IM, Weiderpass E, Campbell PT, Sampson JN, Kitahara $\mathrm{CM}$, et al. Association of leisure-time physical activity with risk of 26 types of cancer in 1.44 million adults. JAMA Intern Med. 2016;176(6):816-25.

40. Lopes-Júnior LC, Bomfim EO, Nascimento LC, Nunes MD, Pereira-daSilva G, Lima RA. Non-pharmacological interventions to manage fatigue and psychological stress in children and adolescents with cancer: an integrative review. Eur J Cancer Care (Engl). 2016;25(6):921-35.

41. Satapathy S, Kaushal T, Bakhshi S, Chadda RK. Non-pharmacological Interventions for Pediatric Cancer Patients: A Comparative Review and Emerging Needs in India. Indian Pediatr. 2018;55(3):225-32.

42. Nunes MD, Bomfim E, Olson K, Lopes-Junior LC, Silva-Rodrigues FM, Garcia de Lima RA, et al. Interventions minimizing fatigue in children/ adolescents with cancer: an integrative review. J Child Health Care. 2018;22(2):186-204. 